\title{
Les Juifs allemands ou la bourgeoisie quand même
}

Tentative d'analyse d'une démarche socio-historique

Das deutsche Judentum oder das ,Bürgertum trotz allem'. Ein soziohistorischer

Blick

German Jewry or the 'Bourgeoisie Anyway': a Socio-Historical Approach

Daniel Azuélos

\section{(2) OpenEdition}

\section{Journals}

Édition électronique

URL : https://journals.openedition.org/ceg/7757

DOI : $10.4000 /$ ceg. 7757

ISSN : 2605-8359

Éditeur

Presses Universitaires de Provence

Édition imprimée

Date de publication : 25 septembre 2019

Pagination : 199-210

ISBN : 979-10-320-0234-6

ISSN : 0751-4239

\section{Référence électronique}

Daniel Azuélos, «Les Juifs allemands ou la bourgeoisie quand même », Cahiers d'Études Germaniques [En ligne], 77 | 2019, mis en ligne le 25 mars 2021, consulté le 15 juin 2021. URL : http:// journals.openedition.org/ceg/7757 ; DOI : https://doi.org/10.4000/ceg.7757 


\title{
Les Juifs allemands ou la bourgeoisie quand même
}

\section{Tentative d'analyse d'une démarche socio-historique}

\author{
Daniel AZUÉLOS \\ Professeur émérite à l'Université de Picardie Jules Verne / CERCLL (EA 4283)
}

"Les Juifs allemands ou la bourgeoisie quand même » : ce titre peut être perçu comme une provocation et elle l'est d'une certaine manière, quand on confronte ma thèse bien modeste à d'autres travaux théoriquement plus ambitieux, mais qui, selon moi, sont assez réducteurs et prennent beaucoup de liberté avec I'histoire. Ma thèse, qui n'est pas exhaustive (je n'ai pas la prétention d'avoir livré une explication définitive et toute analyse historique doit être soumise à débat, cela va de soi), se limite à une interrogation qu'on peut énoncer ainsi : les Juifs allemands étaient au début du XIXe siècle un groupe stigmatisé sur le plan religieux et culturel, et économiquement marginalisé. On peut affirmer sans trop exagérer que l'ascension économique et sociale de la communauté judéo-allemande a été remarquable, au point que, sur toute la période que j'étudiais dans ma thèse (de 1800 aux années 1930), le terme d'embourgeoisement me semble adéquat pour définir le phénomène. Cela ne signifie pas que tout le groupe s'est assimilé à la bourgeoisie allemande, mais qu'une partie significative des Juifs allemands, appuyés et accompagnés par leurs associations cultuelles et culturelles et qu'on identifierait aujourd'hui à des "Mittelständler ", s'est approprié les valeurs de la classe bourgeoise allemande en train de se constituer. On pourrait d'ailleurs plutôt parler de "co-effectuation", bourgeoisie majoritaire et bourgeoisie juive construisant presque simultanément un ethos bourgeois fondé sur les valeurs de la Bildung. Le terminus ad quem ne doit pourtant pas être interprété comme la fin d'une épopée qui aurait commencé au début du XIX siècle (on constate les prémisses d'une telle évolution bien avant), mais d'une tendance qui ne s'est pas démentie pendant un siècle et demi. J'ai donc tenté de mettre au jour les modalités de ce que j'ai appelé « l'entrée en bourgeoisie des Juifs allemands ${ }^{1}$ ». Je pense l'avoir fait en essayant d'évacuer les préjugés que je pouvais avoir encore sur cette question, sine ira et cum studio. Je le dis par avance : Max Weber, Georg Simmel, Norbert Elias furent d'une aide précieuse pour mes recherches, même si je ne les ai pas adossées sur leurs seuls travaux.

1. Daniel Azuélos, L’Entrée en bourgeoisie des Juifs allemands ou le paradigme libéral, Paris, PU Paris-Sorbonne, 2005. 
Pour mieux faire ressortir la thèse que je vais développer à grands traits ultérieurement, j'ai donc souhaité la confronter, par contraste, à un travail dont les résultats sont à l'opposé des miens. Pour être plus exact : j'ai évité dans mon travail, dans la mesure du possible, toute subjectivité et j'envisage l'objectivité comme un idéal vers lequel on doit s'efforcer de tendre, jamais un but que l'on peut espérer atteindre. Je vais donc évoquer l'ouvrage d'un historien du judaïsme allemand qui, selon moi, se situe à l'opposé du cadre déontologique que je me suis fixé pour rédiger mon propre ouvrage et dans les limites duquel tout historien sérieux devrait se mouvoir pour ne pas sombrer dans le piège de l'idéologie. L'ouvrage en question est paru en 2016, il a pour titre La fin de la modernité juive. Histoire d'un tournant conservateur ${ }^{2}$. Son auteur, Enzo Traverso, est professeur à I'Université Cornell aux États-Unis. Je résume en quelques lignes la pensée d'un auteur dont les connaissances sur le sujet sont vastes, mais dont l'interprétation des faits, idéologique, contrevient selon moi aux règles minimales de la discipline historique.

Voici sa thèse : les Juifs ont joué un rôle éminent au sein de la modernité occidentale, leur apport critique a été remarquable. Pour Traverso, être critique, c'est remettre en cause les fondements mêmes de la modernité occidentale, c'est être d'extrême gauche et anti-occidental. II utilise pour cela les concepts de Bernard Lazare, "parvenu » et « paria », tels qu'Hannah Arendt les a revisités. Comme minorité, les Juifs ne seraient vraiment intéressants qu'en qualité de parias, ce qui les met selon Traverso dans une situation idéale pour soumettre I'Occident à une critique radicale. Traverso ne peut bien sûr pas nier qu'il existe aussi des Juifs modérés ne s'inscrivant pas dans cette radicalité : ce sont des Juifs qui, selon lui, ont adhéré au paradigme bourgeois et/ou conservateur, ce qui signifie pour lui la même chose. On trouvera dans le camp du "bien" Karl Marx, Rosa Luxemburg, Jacques Derrida, Max Horkheimer, Noam Chomsky ou Judith Butler. Benjamin Disraeli, Henry Kissinger, Raymond Aron, Leo Strauss voire Ernst Cassirer, pour ne citer que les noms les plus importants, appartiennent quant à eux au camp du "mal", c'est-à-dire le camp modéré ou conservateur. Traverso place Freud dans le camp du "bien", ce qui est problématique à mon avis, car Freud était politiquement très conservateur. II s'agit là d'une vieille lubie de certains intellectuels marqués par la pensée de soixante-huit et qui avaient entrepris, dans une sorte de convergence des luttes, de faire une synthèse osée et peu pertinente entre marxisme, structuralisme et psychanalyse. On voit que ces catégorisations n'ont rien d'historique : elles sont purement idéologiques. Donc, les Juifs, dans la seule mesure où ils ressortissent au groupe des parias, ont de l'intérêt pour l'idéologue radical. Traverso va même plus loin : il stipule un retournement spectaculaire après 1945. L'antisémitisme aurait quasiment disparu en Occident après la Deuxième Guerre mondiale. Le culte autour de l'Holocauste serait devenu un signe de ralliement pour les démocraties occidentales et les Juifs, n'étant plus stigmatisés, deviennent, surtout après la fondation de l'État d'Israël, les porte-

2. Enzo Traverso, La fin de la modernité juive. Histoire d'un tournant conservateur, Paris, La Découverte, 2016. 
drapeau des valeurs occidentales, essentiellement conservatrices, le statut de parias étant dorénavant dévolu au peuple palestinien en lutte. L'islamophobie aurait donc supplanté l'antisémitisme en Occident. II convient ici de remarquer que l'antisémitisme d'origine musulmane, bien antérieur au conflit israélopalestinien, ne fait l'objet d'aucune analyse de la part de Traverso qui adopte des schémas tiers-mondistes simplistes déjà en vogue à l'époque de la guerre froide. De plus, il soumet sa thèse de la disparition quasi-totale de l'antisémitisme au moment même où des attentats ciblant la communauté juive et faisant plusieurs morts, et parmi eux des enfants, avaient lieu en France.

J'arrête là l'exposition d'une thèse qui pose de nombreux problèmes méthodologiques. Traverso construit la fiction d'un peuple juif identique à travers les âges (il succombe ici à un essentialisme qu'il ne manque pourtant jamais de reprocher à certains de ses contradicteurs). Si l'on part en effet du principe que l'identité juive n'est pas seulement réductible à la religion, alors il existe autant de cultures juives qu'il a existé ou qu'il existe de diasporas. Dominique Schnapper nous le rappelle dans son dernier ouvrage consacré aux Juifs et à la citoyenneté 3 . La seule définition qui pourrait s'appliquer aux Juifs dans leur ensemble est séduisante par sa simplicité et c'est la rabbine Delphine Horvilleur qui la présente dans un récent article de Libération :

Ce qui agace le plus chez le juif, c'est qu'il est à la fois comme tout le monde et pas comme tout le monde (...). On reproche toujours au juif une chose et son contraire, d'être discret ou trop bling-bling, révolutionnaire ou bourgeois, parasite de la nation ou trop riche. Et d'être un peu trop semblable et un peu trop différent ${ }^{4}$.

Hannah Arendt, qui défendait en son temps la thèse de la normalisation des Juifs, faisait une remarque presque identique, qui met à mal la conception très réductrice de Traverso :

Au hasard de telle ou telle conception générale du monde à laquelle des auteurs juifs adhéraient, on pouvait identifier le judaïsme soit à l'idée de progrès ou au contraire de résistance à toute volonté subversive de changement, soit à l'Aufklärung ou au contraire à la sauvegarde du monothéisme. On pouvait par exemple de la même façon assimiler les Juifs à des prolétaires-nés, les présenter comme les alliés naturels de la bourgeoisie ou comme la plus vieille aristocratie de l'Occident. (...) [Les Juifs] étaient l'incarnation de quelque chose et non point de simples mortels ${ }^{5}$.

Le problème, c'est que Traverso prend la quasi-totalité de ses exemples de la radicalité juive dans la communauté judéo-allemande d'avant-guerre et qu'il impute le retournement conservateur essentiellement à Israël ou bien aux Juifs vivant actuellement aux États-Unis et en Europe, qui n'ont plus qu'un rapport ténu à la communauté judéo-allemande disparue. C'est une incongruité historique, mais en admettant qu'on s'en tienne au groupe des Juifs allemands

3. Dominique Schnapper, La citoyenneté à l'épreuve. La démocratie et les juifs, Paris, Gallimard, 2018.

4. Delphine Horvilleur, «L'antisémitisme n'est jamais une haine isolée, mais le premier symptôme d'un effondrement à venir », Libération, 8 janvier 2019.

5. Hannah Arendt, « Vom Salz der Erde », Aufbau New York 38 (22 septembre 1944), p. 13. 
d'avant 1933, sa thèse parias/parvenus peut être sérieusement mise en doute. Hannah Arendt avait fait usage de cette thèse à l'époque la plus tragique pour les Juifs d'Allemagne et pour les Juifs européens, à un moment où, chassés d'Europe, ils tentaient désespérément de rejoindre des pays où ils pourraient se sentir en sécurité. Le terme de " paria » semblait alors adéquat à leur situation, mais je le conteste pour toute la période qui va du milieu du XIX ${ }^{e}$ siècle à 1930 environ. On a du mal à croire que les Juifs allemands se vivaient comme des parias. Norbert Elias utilise le terme de " marginaux », " outsiders » en anglais, ce qui semble plus conforme à la réalité. Le terme de " parvenu » n'est pas plus heureux, sauf si l'on définit ainsi toute personne qui aspire à améliorer sa situation économique et sociale et celle de son groupe, ce qui a été le cas pour les Juifs d'Allemagne dans leur ensemble. Si certains des représentants de cette communauté ont joué un rôle critique pendant toute cette période, cette critique n'a pas toujours été radicale et ils ne l'ont pas faite à partir de leur situation plus ou moins imaginaire de « parias ». Je rappellerai simplement la belle phrase de Horkheimer, souvent cité par Traverso pour conforter sa thèse. Parlant des bourgeois allemands, juifs ou non, au rang desquels il se comptait lui-même et qu'il distingue des grands capitalistes, il écrivait :

Les seigneurs du grand capital dévalorisent le plaisir, parce qu'ils sont des barbares, des esclaves de leur entreprise et de leur idéologie, les pauvres méprisent le plaisir pour mieux s'accommoder de leur impuissance. Mais ces bourgeois tardifs ${ }^{6}$ sont justement révolutionnaires parce qu'ils savent ce que signifie le bonheur et aussi que les talents de l'homme finissent par se déprécier et se corrompre quand les conditions ne sont pas favorables ${ }^{7}$.

Enzo Traverso est esclave de son idéologie quand il écrit à propos des nouveaux bourgeois juifs néo-conservateurs dans son chapitre « Les intellectuels entre critique et pouvoir »:

Leur autorité et leur influence ne tiennent ni à leur statut de producteurs de savoirs ni à l'importance de leur œuvre littéraire et scientifique, mais essentiellement à la position stratégique qu'ils occupent au sein de l'industrie culturelle, à l'époque de la réification marchande de l'espace public. Maîtres des codes de la communication, ils se sont parfaitement adaptés aux contraintes de la vidéosphère et savent concilier l'écrit avec la parole et l'image, en faisant de leurs ouvrages des produits de consommation dont la promotion se fait simultanément dans la presse, à la radio et la télévision ${ }^{8}$.

Quelqu'un qui ne connaîtrait pas l'auteur de cette phrase, pourrait tout aussi bien l'attribuer à un intellectuel de la nouvelle droite néo-maurrassienne qui aurait lu Bourdieu!

6. Horkheimer les appelle aussi « outsiders de la bourgeoisie jouissant par anticipation de leurs privilèges comme pour témoigner d'un bonheur qui serait un jour offert au monde entier; ils étaient en quelque sorte la vigie, le gardien d'un point de vue entr'aperçu du haut de leur confort bourgeois ». Max Horkheimer, Gesammelte Schriften, tome 12 (nachgelassene Schriften 1931-1949), Frankfurt a. M., Fischer, 1985, p. 227-232.

7. Max Horkheimer, « Notizen 1935 », ibid., p. 231.

8. Traverso, La fin de la modernité juive, chapitre 3, p. 34. 
Face aux élucubrations d'un idéologue, je proposerai une thèse plus équilibrée: c'est bien tout au long du XIXe siècle le modèle bourgeois allemand, alors qu'il était lui-même en gestation, qui s'est imposé d'emblée aux Juifs allemands comme modèle de référence. On a parlé à cet égard de " symbiose judéo-allemande » ou de "dialogue judéo-allemand », concepts que je n'introduis ici qu'avec une grande prudence. La communauté juive d'Allemagne a éprouvé plus de difficultés que dans d'autres pays, comparables par leur structure socio-économique, à accéder à la pleine citoyenneté. Et pourtant : à la fin du XVIII ${ }^{e}$ siècle et au début du XIX siècle, un certain nombre de territoires allemands (dont la Prusse et l'Autriche des Habsbourg) ont joué un rôle de pionniers pour l'émancipation des Juifs : qu'on songe aux édits de tolérance de Joseph II, à la politique favorable aux Juifs de Wilhelm von Humboldt et Karl August von Hardenberg, ou encore au traité du haut fonctionnaire prussien Christian Wilhelm Dohm, paru en 1781, Über die bürgerliche Verbesserung der Juden, qui inspira l'Abbé Grégoire. Mais la politique régressive du Congrès de Vienne a freiné, sur les territoires des pays de la Sainte-Alliance, le mouvement émancipateur, quand elle n'a pas rétabli des statuts antérieurs, défavorables aux Juifs. Ce n'est qu'assez tardivement que la plupart des États allemands adoptèrent des législations garantissant l'égalité confessionnelle, la Prusse fermant la marche en 1869.

On voit bien là ce qui est au cœur du dilemme de l'assimilation des Juifs à la nation allemande : l'entrée en bourgeoisie des Juifs allemands a précédé leur pleine accession aux droits liés à la citoyenneté, mais il s'est trouvé aussi que la bourgeoisie allemande, au contraire des bourgeoisies anglaise ou française, n'a jamais véritablement touché les dividendes politiques qu'elle était en droit d'attendre compte tenu de ses succès économiques. Ainsi l'obstacle qui se dressait devant les Juifs d'Allemagne en quête d'émancipation était-il particulièrement difficile à franchir. La bourgeoisie qui leur a fourni ses principaux modèles de socialisation était elle-même dans une situation de grande fragilité politique. Elle n'avait pas réussi, au contraire de ce qui arriva en France ou en Angleterre, à faire en sorte qu'on l'identifiât non seulement à la liberté, mais aussi à la nation, thème autrefois révolutionnaire dont le Reich conservateur wilhelminien s'était emparé. Ainsi en est-on arrivé au paradoxe suivant : au XIXe siècle, siècle par excellence du triomphe de la bourgeoisie, la minorité juive la plus active et la plus prospère d'Europe occidentale s'appliquait à modeler son comportement sur la bourgeoisie la plus dynamique du continent, qui avait dû abandonner au pouvoir réactionnaire incarné par Bismarck, puis par le régime personnel de Guillaume II, une grande part de ses prérogatives dans le domaine politique. On peut dire finalement qu'il n'y a jamais eu en Allemagne de déficit en culture bourgeoise. II faudrait plutôt parler de trop plein. La bourgeoisie allemande a donné naissance à un mode d'être et de pensée qui a essaimé dans toutes les couches de la société. Ces valeurs étaient censées être incarnées de façon idéal-typique par une classe aux contours assez flous, baptisée « Bildungsbürgertum », et dont on ne trouve nulle part ailleurs en Europe l'équivalent. Et c'est peut-être autour de cette anomalie allemande que s'est noué le sort d'autant plus précaire de la minorité 
juive qu'elle s'est adossée à une bourgeoisie allemande extrêmement fragilisée, politiquement et socialement.

L'appétence des Juifs d'Allemagne pour la culture majoritaire de leur pays et leur attachement aux valeurs du "Bildungsbürgertum » est une réalité indéniable. Mais il y a surtout cette double énigme : le basculement dans la catastrophe après l'assimilation sans doute la plus réussie d'une minorité à la culture majoritaire. La minorité juive, située majoritairement au plus bas de l'échelle sociale au début du XIXe siècle encore, a réussi à se hisser au niveau de la petite et moyenne bourgeoisie à la fin du siècle.

L'assimilation des Juifs allemands a été avant tout une assimilation aux valeurs de la bourgeoisie allemande. Or, il était impossible pour la minorité juive d'adhérer en blocà l'idéologie allemande telle qu'elle se présentait à l'époque wilhelminienne. Nous en avons une preuve indirecte dans le fait que l'antisémitisme se développa et s'amplifia parallèlement au processus d'assimilation. Les Juifs se trouvaient confrontés au dilemme suivant : ils devaient tout de même se plier un tant soit peu à l'idéologie majoritaire, ou tout au moins composer avec elle, car cela conditionnait malgré tout l'acceptation de la majorité ou sa relative tolérance. Pour ce faire, il convenait de s'arracher au holisme de la communauté d'origine, et cela n'était justement pas possible par un simple transfert du paradigme "juif" vers le paradigme "allemand". II a fallu une médiation : ce furent les Lumières allemandes, ou plutôt l'interprétation qu'en firent les penseurs juifs allemands, de Mendelssohn à Horkheimer ou Hannah Arendt, en passant par la Wissenschaft des Judentums et Hermann Cohen. L'idéologie de la Bildung peut être considérée comme la réponse idiosyncrasique de l'Allemagne au défi de l'individualisme des Lumières occidentales. Le judaïsme allemand s'est bien évidemment transformé sous l'influence des Lumières. Les Juifs allemands participèrent, au même titre que d'autres penseurs allemands, à la construction de l'idéologie de la Bildung, sans se préoccuper de savoir si on leur déniait ou non la capacité de gérer ce bien spirituel commun, pour paraphraser une formule célèbre de Moritz Goldstein ${ }^{9}$. La plupart des penseurs juifs étaient en connivence avec la Bildung allemande, qu'ils percevaient comme le modèle idéal dans lequel ils se coulèrent sans trop de difficulté et qu'ils contribuèrent aussi à façonner, tout en étant parfois en décalage ou en retrait par rapport à lui.

Mendelssohn, en disciple de Lessing, n'a pu convaincre ses interlocuteurs allemands, de Lavater à Humboldt, qu'il n'y avait pas d'incompatibilité entre un judaïsme, même réformé, et la croyance en la raison universelle. L'offre d'émancipation présentée par Humboldt, lui-même disciple de Dohm, était problématique, car il maintenait la possibilité d'une adhésion de tous à un horizon communautaire chrétien de nature holiste, sous la forme, certes, d'un projet à échéance indéterminée. Mendelssohn, lui, s'accrochait à la nécessité du pluralisme religieux. La proposition de Humboldt ne pouvait de toute façon se concevoir qu'à la seule condition que le pan libéral qui sous-tendait toute sa

9. Moritz Goldstein, « Deutsch-jüdischer Parnass », Der Kunstwart 25, 1912, p. 283. 
réflexion d'homme politique, investi de responsabilités au sein du gouvernement de Prusse, ne s'effondrât pas. Car alors intervenait le risque, bien perçu par Mendelssohn, de fusion ou d'englobement dans un tout non réductible à l'espace politique. Et c'est bien ce qui a fini par arriver dans une Allemagne fascinée par la soumission au holisme communautaire, sans jamais être capable tout au long du XIX siècle de faire droit à une approche rassérénée, non crispée de la réalité sociale et politique.

Plus on avance dans le siècle, plus se pose de façon aiguë le problème de la place des Juifs au sein de la nation allemande et de leur adhésion aux valeurs de la germanité. Les Juifs allemands commençaient à s'intégrer à une bourgeoisie dont ils partageaient la plupart des valeurs et les fruits de ses succès dans les domaines économique, scientifique et culturel, ils mettaient en place des institutions communautaires souvent calquées sur les institutions allemandes, mais ils se trouvèrent bientôt en porte-à-faux par rapport à une nationalisation du discours majoritaire de la Bildung, qu'ils essayèrent malgré tout de transposer avec les difficultés que l'on sait. La première tentative d'historisation de la culture juive, sous l'influence de la philosophie hégélienne, avait été l'œuvre du Verein für Cultur und Wissenschaft der Juden, dont Heine avait été l'un des membres fondateurs aux côtés d'Eduard Gans et de Leopold Zunz. La deuxième phase de I'institutionnalisation de la Wissenschaft des Judentums, qui va de 1848 à la fin du siècle, fut plus fructueuse sur le plan de l'adaptation de la culture juive mémorielle au discours majoritaire. Les Juifs allemands de la période wilhelminienne adhérèrent d'emblée à l'individualisme substantiel de la Bildung, sans en être complètement prisonniers. Au fond, ils réinterprétaient l'idéologie de la Bildung en l'infléchissant dans un sens plus occidental, plus proche des origines (les Lumières françaises et allemandes ou même anglaises). C'est là tout le paradoxe : pour coller le mieux possible au subjectivisme profond de la Bildung, ils n'avaient d'autre choix que de l'inscrire dans une conception plus large, plus respectueuse de leur différence. Le dilemme était insurmontable. Jouer complètement le jeu des valeurs occidentales était suicidaire, car la communauté juive se plaçait alors délibérément dans le camp de l'adversaire, à une époque d'exacerbation des passions nationales. Jouer à fond les valeurs propres du judaïsme en refusant de s'adosser aux valeurs allemandes n'avait pas non plus de sens, dès lors que la minorité avait fait le choix de s'assimiler à la bourgeoisie et que la sécularisation était devenue un processus irréversible. II ne restait plus aux Juifs allemands qu'à assumer cette voie étroite qui était en même temps transformation et adaptation de la Bildung et qui était en ce sens ambivalente qu'elle avait toujours en ellemême ce contre quoi elle protestait.

Les Juifs allemands n'échappèrent pas tout à fait à la tentation nationaliste : les exemples ne manquèrent pas, à l'instar du philosophe Hermann Cohen qui, à l'aube de la première guerre mondiale, esquissa la thèse d'une proximité profonde entre judaïsme et germanité. Pour autant, le judaïsme allemand renoua très vite, à quelques exceptions près, avec un courant de pensée libéral et démocratique qui l'avait toujours irrigué souterrainement, s'identifiant plus que tout autre groupe en Allemagne avec la nouvelle République issue de la défaite, s'isolant de plus en 
plus et se coupant du courant majoritaire au fur et à mesure que la crise radicalisait les partis de la droite traditionnelle et une gauche à laquelle les Juifs n'adhérèrent bien souvent que par raccroc et sans conviction. L'historien américain Peter Gay résume bien le positionnement politique des bourgeois juifs sous la République de Weimar quand il parle de son père, Moritz Fröhlich : "C'était un bourgeois plein d'ambition, un fidèle partisan du SPD, ce qui n'était pas une preuve de son radicalisme, mais témoignait du fait que ce parti avait gagné en respectabilité ${ }^{10}$. »

Cependant, les relations entre majorité et minorité ne se résument pas à l'adaptation ou à la transposition d'une idéologie : elles s'inscrivent aussi dans un espace et une histoire, elles relèvent d'un processus dynamique qui confère au phénomène étudié toute sa singularité et son originalité. L'interprétation par les Juifs allemands de l'idéologie de la Bildung ne prend tout son sens que si l'analyse de l'interaction entre majorité et minorité est prise dans un système configurationnel qui, même s'il est toujours fluctuant et mouvant sur le moyen et le long terme, peut donner sur le court terme l'illusion de l'immobilité. On peut ainsi essayer d'appréhender la place singulière d'un groupe attaché par son habitus et son idéologie à la classe la plus dynamique de la société, mais en même temps stigmatisé pour s'être, croyait-on, peut-être trop bien coulé dans ce moule bien adapté pour affronter les défis de la modernité.

On constate avec Norbert Elias qu'il n'y a jamais eu en Allemagne d'adéquation entre le rôle social et culturel de la minorité et la place qu'elle aurait pu revendiquer dans les domaines politique et institutionnel, au contraire de ce qui s'est passé en France et en Angleterre. En examinant l'évolution de la bourgeoisie allemande et l'inscription de la minorité juive dans la problématique de la Bürgerlichkeit, il est possible d'y voir plus clair sur les raisons d'un parcours fructueux mais aussi d'un échec tragique, de montrer pourquoi la conjonction entre un mode d'existence, ses représentations, ses rêves, son idéologie et une communauté qui s'y reconnaissait au moins en partie, a été aussi fructueuse et a pu en même temps précipiter sa chute.

Le processus que j'ai appelé « entrée en bourgeoisie des Juifs allemands » n'a pas été précédé par la naissance d'une bourgeoisie que la minorité souhaitait rejoindre après qu'elle se fut constituée en classe autonome. En fait, il semblerait plutôt que c'est au fur et à mesure que s'élaborait, depuis l'époque des Lumières, l'idéologie de la Bürgerlichkeit, que les Juifs allemands s'approprièrent ces normes comportementales et ces valeurs qui les confortaient dans leur volonté d'intégration et d'ascension. II y a eu renforcement réciproque, consolidation de l'habitus initial déjà préparé par l'idéologie des Lumières au contact de la bourgeoisie, comprise comme culture et non comme classe sociale. La minorité juive faisait sienne une culture bourgeoise qui était tout juste en train d'inventer son propre canon et ses propres normes. On ne comprendrait pas autrement la facilité avec laquelle ces normes et ces valeurs ont été intériorisées, s'il avait fallu qu'elles passent par la médiation d'une classe déjà constituée. C'est dans la mesure

10. Peter Gay, Meine deutsche Frage. Jugend in Berlin. 1933-1939, München, C. H. Beck, 1999, p. 35. 
même où elles étaient immédiatement universalisables qu'elles trouvèrent un terreau pour prospérer.

Cette capacité à construire sa propre vie, qu'on a pu décrire chez des individus exceptionnels (Moses Mendelssohn, Salomon Maïmon), a été par la suite reprise à son compte par le groupe tout entier dont les instances communautaires se sont lancées dans un programme d'éducation ambitieux qui s'émancipait de l'emprise rabbinique traditionnelle pour se couler dans les normes de la Bildung. L'historienne Simone Lässig évoque l'« invention d'une religion bourgeoise ${ }^{11}$ » qui est sensiblement différente de celle du groupe majoritaire en ce que la minorité s'est attachée à en faire bénéficier également, par tout un système de bourses, les couches les moins favorisées de la communauté. D'autres éléments sont venus renforcer ces programmes d'éducation : de sa propre initiative, le groupe juif, à travers ses institutions, a imposé non sans mal aux rabbins, orthodoxes comme libéraux, des services religieux en langue allemande. Les modifications du rituel (introduction de l'orgue et, pour certaines rares synagogues, mise en place de l'office religieux le dimanche, etc.), qui pouvaient être condamnées par ailleurs par ceux qui craignaient de voir se dissoudre le dogme dans une religion de la raison qu'avait en son temps appelée de ses vœux Salomon Maïmon ${ }^{12}$, avaient pour but affirmé de soumettre le groupe à un canon de valeurs et de vertus bourgeoises adossées à la Bildung et à la Bürgerlichkeit. Lässig souligne au passage que les instances religieuses du judaïsme allaient beaucoup plus loin en la matière que les instances catholiques ou protestantes, qui avaient davantage tendance à exclure les éléments profanes du cadre religieux. Tout cela n'aurait sans doute pas eu le même effet s'il n'y avait eu un accompagnement par un réseau d'institutions profanes et par une presse pléthorique. II n'y a donc pas eu simplement transposition d'un modèle préexistant bourgeois-allemand, mais bien plutôt adaptation et transformation de ce même modèle.

Si j'ai postulé qu'il a existé quelque chose comme une affinité élective entre nouvelle bourgeoisie et minorité juive, ce n'est pas parce que la composition sociologique du groupe juif coïncidait précisément avec une classe bourgeoise aux contours préalablement définis. Le caractère de marginalité et de mobilité de la minorité juive l'apparentait non pas à la classe bourgeoise telle qu'on la définit généralement, mais à toute une série de normes comportementales et d'attitudes que l'on peut qualifier de bourgeoises. Au contraire des classes traditionnelles, la noblesse, la paysannerie, la bourgeoisie des corporations, l'univers culturel de la nouvelle bourgeoisie, telle que Max Weber la définissait dans L'Éthique protestante et l'esprit du capitalisme ${ }^{13}$ et qui va s'affirmer tout au

11. Simone Lässig, Jüdische Wege ins Bürgertum. Kulturelles Kapital und sozialer Aufstieg im 19. Jahrhundert, Göttingen, Vandenhoeck \& Ruprecht, 2004, p. 660.

12. Voir Daniel Azuélos, «Aux origines de l'appétence juive pour la Bildung allemande : Salomon Maïmon (1753-1800) raconté par lui-même », in Maiwenn Roudaut (dir.), Retours sur l'émancipation des Juifs en Europe au XVIII' siècle, Bordeaux, PU Bordeaux, 2017, p. 91-106.

13. Max Weber, Die protestantische Ethik und der Geist des Kapitalismus, Bodenheim, Athenäum Hain Hanstein, 1993. 
long du XIX siècle, était censé concerner l'humanité dans son acception la plus large : la Bildung devenait Menschenbildung.

La bourgeoisie était vouée à devenir une sorte de classe universelle, susceptible d'englober un jour toutes les autres classes de la société. L'historien Lothar Gall parle d'« allgemeiner Stand » par excellence ou d'« allgemeiner Stand der gebildeten Menschen ». On trouve aussi sous sa plume l'expression de " klassenlose Bürgergesellschaft der Freien und Selbstständigen ${ }^{14}$ ». Mais la bourgeoisie ne peut qu'être piégée par le caractère utopique de son message universaliste. Car au moment même où elle le prend au sérieux, elle a tendance à se refermer, dans la mesure où le principe d'individualisme et d'autonomie qui fonde son idéologie conduit ses membres à se livrer une concurrence acharnée pour survivre et les entraîne dans cette spirale matérialiste décrite par Max Weber. La gageure n'a jamais pu être tenue, le pari utopique de l'égalisation des chances par l'accès de tous à la culture et au savoir s'est révélé impossible. II n'en reste pas moins que la dimension utopique de son idéologie a survécu à la fossilisation sociale, conséquence des combats menés sur les deux fronts politiques qui l'ont opposée à la noblesse jusqu'en 1914 ainsi qu'au prolétariat, qui n'a cessé de s'affirmer tout au long de la période wilhelminienne. La minorité juive s'est donc tout naturellement rangée, non pas sous la bannière de la bourgeoisie constituée en classe sociale, mais sous celle de la Bürgerlichkeit porteuse des valeurs universelles héritées des Lumières.

J'ai appuyé ma démonstration sur quelques exemples tirés de la vie économique et sociale. Mais il me semble intéressant de vérifier ces hypothèses par un autre biais. Il peut arriver que des œuvres de fiction, qu'elles soient écrites par des génies ou des auteurs de moindre envergure, renvoient une image plus authentique de la réalité que de longs traités socio-économiques et leur appareil statistique. C'est avec surprise que j'ai découvert que certains romans écrits par des écrivains juifs reprenaient dans leur fiction, sans que les auteurs en aient véritablement conscience, des thèmes que j'avais analysés et qui ont structuré l'imaginaire des Juifs allemands pendant toute cette période. Beaucoup de romans pourraient être étudiés suivant le couple individualisme/holisme ou modernité/tradition, que j'ai mis au jour dans mon étude. Je pense notamment à Der Pojaz ${ }^{15}$ de Karl Emil Franzos, publié en 1905. Ce sont cependant deux autres romans écrits par des auteurs juifs, Georg Hermann et Wilhelm Speyer, que je me suis proposé de comparer et de confronter à ma problématique ${ }^{16}$. L'un situe son action (en fait il s'agit d'un diptyque, Jettchen Gebert ${ }^{17} /$ Henriette Jacoby ${ }^{18}$ ) à l'époque du Vormärz à Berlin, I'autre roman Das Glück der Andernachs ${ }^{19}$ englobe une période qui va de la fin du règne de Guillaume ler à l'avènement de Guillaume II et au début de l'ère bismarckienne. Les romans de Georg Hermann ont été

\footnotetext{
14. Lothar Gall, Bürgertum in Deutschland, Berlin, Siedler, 1989.

15. Karl Emil Franzos, Der Pojaz, eine Geschichte aus dem Osten, Stuttgart, Cotta, 1907.

16. Azuélos, L'Entrée en bourgeoisie, p. 339-369.

17. Georg Hermann, Jettchen Gebert, Berlin, Fleischel, 1910.

18. Georg Hermann, Henriette Jacoby, Berlin, Fleischel, 1908.

19. Wilhelm Speyer, Das Glück der Andernachs, Zürich, Micha, 1947.
} 
écrits en 1906 et 1909; le roman de Speyer en 1947. Hermann se propose de faire revivre avec nostalgie l'époque la plus riche de promesses de la « symbiose judéoallemande »; Speyer met au jour sur une façade d'apparente réussite économique et d'accomplissement social les lézardes annonciatrices de la catastrophe prochaine.

Le diptyque de Georg Hermann et le roman de Wilhelm Speyer illustrent, chacun à sa manière, la tragédie vécue par des bourgeois juifs allemands trompés par une trop grande confiance dans le message universaliste de la Bildung. Certes, ce pari sur les valeurs universelles de l'utopie bourgeoise et du libéralisme n'est jamais remis en cause par les acteurs les plus conscients des deux romans, il est maintes fois réitéré à des moments essentiels dans le développement de la fiction. Tout se passe comme si les deux figures exemplaires de l'existence bourgeoise dans chacun des romans étaient partis en guerre contre une forme d'hybris, de démesure, qui avait fini par menacer l'équilibre tant familial que sociétal. L'un et l'autre s'appliquent, sans succès, à résoudre la difficulté de réaliser une synthèse raisonnable entre modernité et tradition. Le premier, Jason Gebert, le héros de Georg Hermann, tente de conjurer le mal en évoquant Heinrich Heine, figure de la modération, contre Börne l'échevelé, l'apôtre du tout politique. De la même façon, le personnage principal du roman de Speyer, Jakob Ephraim Andernach, revendique, contre son contradicteur et néanmoins ami, Christian Adam Rauch, un bourgeois libéral allemand dans la tradition de 1848, une vision plus sereine et plus dégagée de la chose politique. On retrouve au cœur de ces fictions les interrogations fondamentales qui m'ont servi de fil rouge tout au long de ma réflexion : comment le choix de l'individualisme a-t-il permis à une minorité de s'arracher au holisme de la tradition ? L'individualisme substantiel de la différence (caractéristique de l'idéologie allemande) peut-il coexister avec l'individualisme de l'identité (le mode occidental de l'individualisme) ? La société juive, prise entre deux formes de holisme, le sien propre et celui de son environnement chrétien, n'avait-elle pas vocation à faire pencher la balance du côté de l'individualisme occidental pour faire advenir plus rapidement une modernité qui l'avait propulsée avec profit dans le siècle ? Mais en même temps, l'adhésion sans contrepoids à cette modernité ne s'accompagnait-elle pas d'une perte de substance?

Les personnages de référence des deux romans essaient ainsi désespérément de tenir ensemble les deux bouts de la chaîne, de ne pas céder au vertige d'un holisme en apparence rassurant, mais de refuser en même temps de se plier à la forme la plus extrême de l'individualisme : un matérialisme niveleur et sans âme, dont Max Weber avait été l'un des tous premiers à montrer au début du XXe siècle qu'il risquait d'emporter la mémoire et le fond traditionnel de nos sociétés. Ce n'est pas un hasard si des penseurs juifs issus de cette bourgeoisie libérale, dont j'ai essayé de retracer le parcours, comme Walter Benjamin, Max Horkheimer, Hannah Arendt, Ernst Cassirer et bien d'autres encore, se sont inscrits dans cette tradition.

La bourgeoisie, qui a incarné au plus haut point la modernité avec ses espoirs et ses désillusions, s'est trouvée tout naturellement au centre de toutes les polémiques et est devenue l'objet des rancœurs les plus contradictoires, qu'on 
l'accuse d'avoir freiné le processus de démocratisation ou à l'inverse d'avoir ouvert trop tôt la boîte de Pandore des illusions démocratiques. II n'est pas étonnant de trouver au carrefour de toutes ces contradictions des membres de la minorité juive, tant l'identification avec la tradition la plus authentique des vertus bourgeoises était forte chez la plupart d'entre eux et tant le déclin des valeurs bourgeoises pouvait constituer une menace non seulement pour les libertés en général, mais singulièrement pour la poursuite du processus d'assimilation qui les concernait au premier chef.

Les Juifs allemands ne pouvaient qu'exceptionnellement et seulement au prix de la conversion se couler dans le modèle bourgeois allemand tel qu'il s'était développé sous le Reich wilhelminien. II ne leur était guère possible d'élargir leur champ d'action social en développant des stratégies d'alliance au-delà de leur propre communauté, ni d'avoir l'ambition de coordonner les différentes branches de la bourgeoisie. Ils ne représenteraient en effet jamais qu'un seul courant de la bourgeoisie : celui qui resterait toujours adossé à l'utopie primitive et qui serait le moins enclin aux compromis idéologiques, alors que la bourgeoisie non juive pouvait se déployer dans toutes les directions. Au fond, les Juifs allemands restèrent plus longtemps fidèles, pour des raisons historiques et idéologiques, à un mode d'être bourgeois qui s'était mis en place tout au long du XIX siècle et qu'ils avaient contribué à façonner, mais qui était voué au déclin dans la population générale. Cela a été leur chance pendant un siècle et demi, mais cela précipita aussi leur chute. 